\title{
Note on the short-period variability of V521 Cas in NGC 7789
}

\author{
P. Van Cauteren ${ }^{1}$, P. Lampens ${ }^{2}$ and A. Pigulski ${ }^{3}$ \\ ${ }^{1}$ Beersel Hills Observatory (BHO), Laarheidestraat 166, 1650 Beersel, Belgium \\ (paulvancauteren@skynet.be) \\ 2 Koninklijke Sterrenwacht van België, Ringlaan 3, 1180 Ukkel, Belgium \\ (patricia.lampens@oma.be) \\ ${ }^{3}$ Wrocław University Observatory, Kopernika 11, 51-622 Wrocław, Poland \\ (pigulski@astro.uni.wroc.pl)
}

\begin{abstract}
Recent observations of V521 Cas in the open cluster NGC 7789 do not confirm that the star is a $\delta$ Scuti variable (within the observational limits).
\end{abstract}

V521 Cas (mag 14.4) was photoelectrically discovered as a variable member of the open cluster NGC 7789 by Danziger (1971) in 1968. Based on 16 measurements obtained on a single night, a period of about 4 hours and a full amplitude of $0.1 \mathrm{mag}$ - possibly variable-were derived. Based on these parameters, the star (also known as Küstner 573 in NGC 7789) was classified as a new $\delta$ Scuti star (Rodríguez et al. 2000). Breger (1983) re-determined the period found by Danziger (1971) using the method of Fourier analysis and found a best value of $0.147 \pm 0.037 \mathrm{~d}$ : he recommended to adopt a value of $0.15 \mathrm{~d}$. Jahn et al. (1995) observed the cluster on four nights in 1991 and discovered 15 new variable stars, of which one was a $\delta$ Scuti star, but they did not mention V521 Cas. Mochejska and Kałużny (1999) extensively searched for variable stars in NGC 7789 in the magnitude range 14 to 20: on five consecutive nights they obtained 22.5 hours of data. This resulted in over 10.000 stars being measured. However, they did not mention V521 Cas either. Furthermore, Zhang et al. (2003) do not list V521 Cas among the variable stars they detected in the $1^{\circ} \times 1^{\circ}$ field observed around the cluster position.

We re-observed V521 Cas on two successive nights (15/16 and 16/17) in October 2003 at BHO with the 0.4-m Newtonian telescope equipped with a 
ST10XME CCD camera and a Bessell V filter: $101 \mathrm{CCD}$ frames (after stacking per 10) were collected. The mean standard deviation on the differences $\Delta \mathrm{V}$ (V521 Cas - comparison star) for two nights was 15.5 mmag. After Fourier analysing these data with Period98 (Sperl 1998), we concluded that no variations with an amplitude larger than 14 mmag (i.e. $4 \times$ the mean noise level of $3.5 \mathrm{mmag}$ in the periodogram ) could be detected in the 2003 data.

Since these data are too scarce to draw any firm conclusion on the star's variability, we also re-analysed the KPNO data obtained by Mochejska \& Kałużny (1999). Except for a small trend over the 5 days we found no variations. In particular, no short-period variability with an amplitude exceeding 5 mmag has been found (Fig. 1).

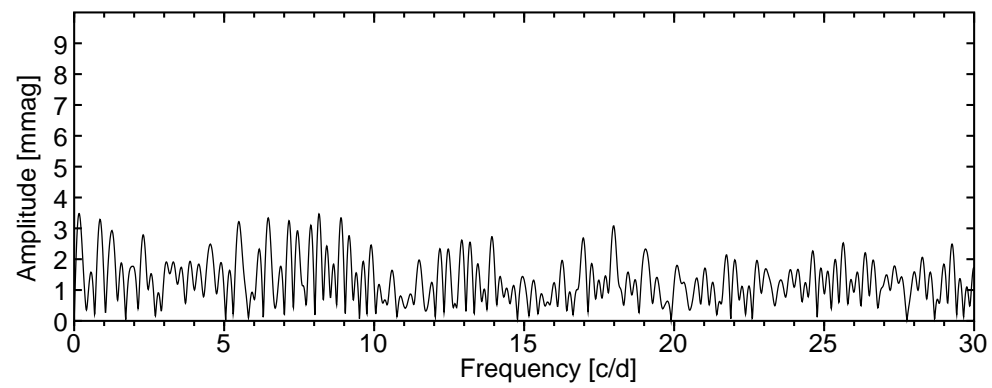

Figure 1: Fourier periodogram for the 1992 KPNO data of V521 Cas.

In conclusion, the observations acquired during the last decade (in 1991, $1992,2000,2003)$ show no evidence for the short-period variability of V521 Cas above the detection threshold. There are two possibilities: (a) a less probable one: its actual amplitude decreased below the detection threshold of several mmag; and (b) a more probable one: the star is (was) constant and the shortperiod variability found by Danziger (1971) is caused by the observational errors or some other observational selection effect. Note also that the star has a nearby faint companion, and that the stars are not easily resolved with a photoelectric photometer.

Acknowledgments. We are grateful to B. Mochejska and J. Kałużny for providing the NGC 7789 data obtained at Kitt Peak National Observatory. P. Van Cauteren is grateful to the Royal Observatory of Belgium for putting at his disposal material acquired by project G.0178.02 of the Fund for Scientific Research (FWO) - Flanders (Belgium). Part of the data were acquired with equipment purchased thanks to a research fund financed by the Belgian National Lottery (1999). 


\section{References}

Breger, M. 1983, IBVS 2393

Danziger, I.J. 1971, PASP 83, 84

Jahn K., Kałużny, J. and Ruciński, S.M. 1995, A\&A 295, 101

Küstner, F. 1923, Veröff. Univ.-Sternw. Bonn 18

Mochejska B.J. and Kałużny, J. 1999, Acta Astronomica 49, 351 (MK)

Rodríguez, E., López-González, M.J. and López de Coca P. 2000, A\&AS 144, 469

Sperl, M. 1998, Comm. in Asteroseismology 111, 1, ed. M. Breger (Vienna)

Zhang, X.B., Deng, L.C., Xin, Y. and Zhou, X. 2003, ChJA\&A 3, 151 